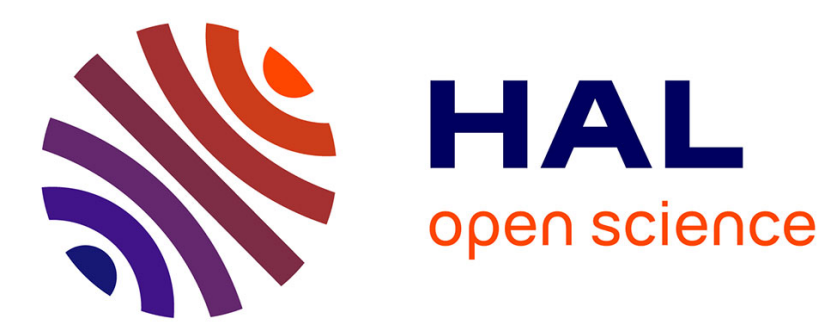

\title{
Quiet broadband light
}

Hugues Guillet de Chatellus, O Jacquin, O Hugon, E Lacot

\section{To cite this version:}

Hugues Guillet de Chatellus, O Jacquin, O Hugon, E Lacot. Quiet broadband light. Physical Review Online Archive (PROLA), 2014, 90, pp.1050-2947/2014/90(3)/033810(6). 10.1103/PhysRevA.90.033810 . hal-01102193

\section{HAL Id: hal-01102193 https://hal.science/hal-01102193}

Submitted on 12 Jan 2015

HAL is a multi-disciplinary open access archive for the deposit and dissemination of scientific research documents, whether they are published or not. The documents may come from teaching and research institutions in France or abroad, or from public or private research centers.
L'archive ouverte pluridisciplinaire HAL, est destinée au dépôt et à la diffusion de documents scientifiques de niveau recherche, publiés ou non, émanant des établissements d'enseignement et de recherche français ou étrangers, des laboratoires publics ou privés. 


\title{
Quiet broadband light
}

\author{
H. Guillet de Chatellus, O. Jacquin, O. Hugon and E. Lacot* \\ CNRS/Univ. Grenoble 1, Laboratoire Interdisciplinaire de Physique, UMR 5588, Grenoble, F-38041, France
}

(Dated: May 9, 2014)

\begin{abstract}
The interference of waves evenly separated in frequency generates a periodic signal, whose intensity fluctuations depend on the phases of the individual waves. A fundamental question in telecommunications and acoustics is the minimization of the Peak to Average power Ratio (PAR): for a given spectrum, how to arrange the phases of the individual frequency components to ensure minimal intensity fluctuations of the resulting signal? For a flat spectrum a near-optimal solution is brought by the so-called Newman phases, commonly used in acoustic and radio waves. Here we transpose this property into the optical domain and prove the possibility to suppress the intensity fluctuations resulting from intermodal beating of a broadband comb of optical frequencies, whose phases are set according to a generalization of the Newman phases. We demonstrate experimentally a broadband laser, whose intensity contrast (defined as the standard deviation-to-mean ratio) is reduced down to 0.54 as compared to 1 when the phases are set randomly.
\end{abstract}

PACS numbers: 42.60.By, 42.60.Pk, 42.60.Fc, 42.25.Kb

Minimizing the fluctuations of a signal is a problem common to acoustics, radar, sonar and wireless telecommunications where it is known as the Peak to Average power Ratio (or PAR) problem: for a given discrete spectrum how to arrange the phases of the spectral components, to achieve the smallest peak factor, that is to minimize the intensity fluctuations resulting from intermodal beating? This question is particularly crucial in communications schemes based on Orthogonal Frequency Division Multiplexing (OFDM) and in data processing, where the amplification of strongly fluctuating signals results in severe intermodulation nonlinear distortion and spectral spreading [1]. Interestingly this simple problem is still open and no systematic optimal solution has been provided yet $[2-5]$. However it turns out that particular choices of the phases lead to relatively low peak factors: among them the Rudin-Shapiro phases enable small peak factors but in the specific case of $2^{p}$ frequency components $[6,7]$. This restriction is lifted by the so-called Schroeder phases which enable remarkably low peak factors with no condition on the number of modes nor on their relative amplitude. Schroeder phases were deduced from considerations on the asymptotic spectra of certain frequency-modulated signals [8]. Assume a periodic signal expressed as the Fourier series $s(\theta)=\sum_{n=1}^{N} \sqrt{p_{n} / 2} \cos \left(n \theta+\varphi_{n}\right)$ where $p_{n}$ is the relative power of the $n$ harmonic $\left(\sum_{n=1}^{N} p_{n}=1\right)$. When the phases are set according to $\varphi_{n}=\varphi_{1}-\sum_{l=1}^{n-1}(n-l) p_{l}$ the peak factor is strongly reduced and the resulting signal appears very similar to a chirped frequency-modulated wave displaying constant amplitude and monotonous instantaneous frequency $[8,9]$. In the case of a flat spec$\operatorname{trum}\left(p_{n}=1 / N\right)$ the phases reduce to $\varphi_{n}=\varphi_{1}-\frac{\pi n^{2}}{N}$ and match the set of phases introduced earlier by Newman in the frame of polynomials extremal problems $[10,11]$ (Fig. 1). Since then, Newman phases have been largely popularized in various domains where the PAR must be mini-
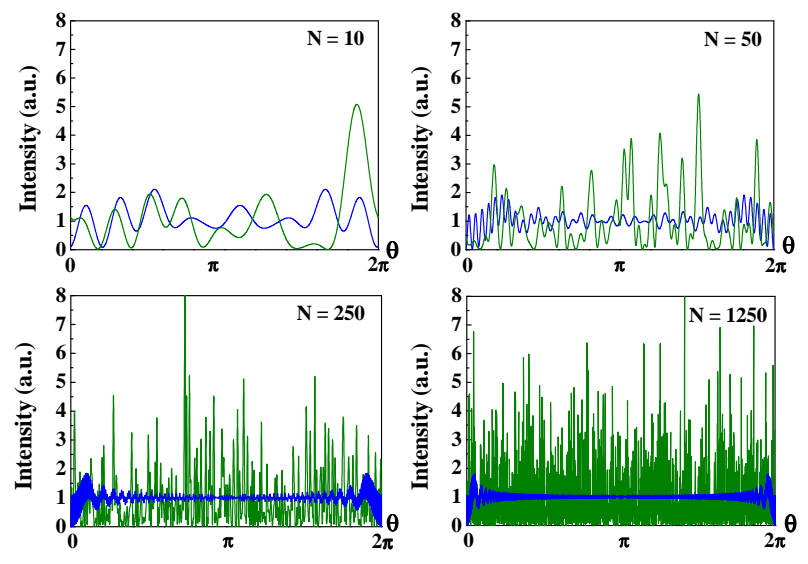

FIG. 1. Reduction of the intensity fluctuations of a flat comb of $N$ optical frequencies arranged according to the Newman phases. The evolution of $I(\theta)=\left|\sum_{n=1}^{N} e^{-i n \theta+i \varphi_{n}}\right|^{2}$ with the dimensionless time $\theta$ is computed for both Newman phases (i.e. $\varphi_{n}=-\pi n^{2} / N$, in blue) and random phases (in green) for different values of $\mathrm{N}(10,50,250$ and 1250). The degree of second order coherence at zero delay $g^{2}(0)$ (see text) is equal to $2.0 \pm 0.1$ in all four cases of random phases, while it is equal respectively to $1.21,1.09,1.04$ and 1.02 in the cases of the Newman phases.

mized, from acoustics [12-14] and adaptive noise control [15] to radar [16] and wireless communications [17].

In classical optics, the inverse problem is much more common: the maximization of the peak power, e.g. by mode-locking techniques, enables to generate trains of ultrashort pulses to reach non-linear regimes or to provide a sharp temporal selectivity for ultrafast applications. Consequently the question of minimizing the (classical) fluctuations of multimode light by a specific arrangement of the phases of the individual frequency components, has been largely ignored. However this problem could 
bring solutions to reduce the distortion in optical amplifiers, and to avoid deleterious non-linear effects in fibers such as spectral broadening and non-linear coupling between distinct frequencies. Nevertheless notice that the transposition of the results obtained so far for radio- or acoustic waves to optics, imposes certain requirements on the comb of optical frequencies. First the effects of quantum noise should be negligible with respect to the classical inter-mode fluctuations and second, the relative amplitudes of the individual frequencies should remain constant at the timescale of the measurement.

In this paper we provide for the first time to our knowledge, the demonstration of a broadband laser delivering a large number of equidistant optical frequencies, whose intensity fluctuations resulting from intermodal beatings are significantly lowered by a specific arrangement of the phases. The manuscript is structured as follows: in the first part after discussing the measurement of the intensity fluctuations of light, we generalize the concept of the Newman phases and exhibit many other specific sets of quadratic phases enabling to reduce the intensity fluctuations of a flat comb of optical frequencies. In the second part we report the experimental demonstration of a Ti:Sa laser containing about 2500 frequencies whose phases have been set according to the generalized Newman phases. We demonstrate a reduction of the contrast of the intensity fluctuations by a factor of 2 , compared to the case where the phases are set as random.

Measurement of the intensity fluctuations of broadband light In acoustics or radiowave communications several estimators of the intensity fluctuations can be provided, like the crest factor, the peak-to-peak amplitude or the PAR [12]. In optics the usual way of quantifying the intensity fluctuations is the degree of second-order coherence (or second-order autocorrelation function) defined as $g^{(2)}(\tau)=\frac{\langle I(t) I(t+\tau)\rangle}{\langle I(t)\rangle^{2}}$ where $I(t)$ is the intensity of the optical field and $\langle>$ denotes the average over time. The value of $g^{(2)}(\tau=0)$ gives a particularly simple estimation of the intensity fluctuations: for instance perfectly coherent light and phase-modulated light display no (classical) intensity fluctuations which corresponds to $g^{(2)}(0)=1$. On the contrary a set of $N$ equidistant optical frequencies with constant phases as in mode-locked lasers, show maximum intensity fluctuations and $g^{(2)}(0)=N$. When the phases are chosen at random the behavior is similar on average to chaotic light and $g^{(2)}(0)=2$ in the limit where $N$ is large [18]. The degree of second order coherence is also directly linked to the contrast of the intensity defined as $C=\frac{\sqrt{\left\langle I^{2}\right\rangle-\langle I\rangle^{2}}}{\langle I\rangle}=\sqrt{g^{(2)}(0)-1}$. Experimentally the technique of second harmonic generation (SHG) enables to record $g^{(2)}(\tau)$ : the interferometric autocorrelation (IAC) trace recorded by measuring the SHG at the output of a two-beam interferometer and averaged over the fringes, is proportional to $g^{(2)}(0)+2 g^{(2)}(\tau)$ where $\tau$ is the time-delay of the interferometer. For comparison
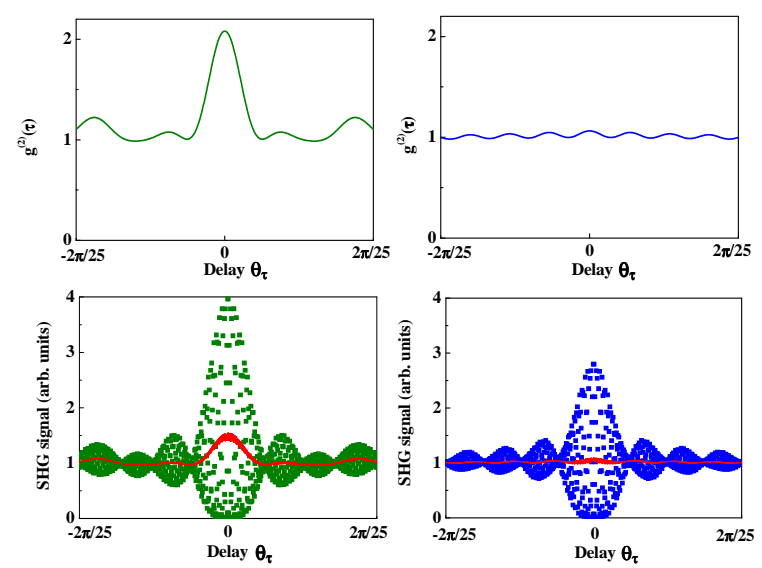

FIG. 2. Top: comparison of the degree of second order coherence $g^{(2)}(\tau)$ in the case of a flat spectrum of 100 equidistant optical frequencies with phases set as random (green, left) and according to the Newman phases $\varphi_{n}=-\pi n^{2} / N$ (blue, right). Bottom: corresponding normalized IAC $\left|\left(E(\theta)+E\left(\theta+\theta_{\tau}\right)\right)^{2}\right|^{2}$. The red trace is the average over the fringes. The peak-tobaseline ratio of the fringes are close to $4: 1$ and $8: 3$ in the case of the random and Newman phases respectively while the peak-to-baseline ratio of the average traces are $3: 2$ and $1: 1$ respectively.

theoretical plots of the degree of second order coherence and the IAC trace in the case of random and Newman phases are plotted on Fig. 2.

Generalization of the Newman phases We now demonstrate a generalization of the Newman phases. Consider a set of $N$ equidistant optical frequencies labelled by $n$ with amplitudes equal to $E_{0} h(n)$ and separated by the angular frequency $\omega_{s}(h$ vanishes outside the domain $0 \leq n \leq N-1$ ). In link with the Newman phases, we consider a quadratic dependence of the phases. The resulting electric field is:

$$
\mathfrak{E}_{\varphi}(t)=E_{0} \sum_{n} h(n) e^{-i\left(\omega_{0}+n \omega_{s}\right) t} e^{i \frac{n^{2}}{2} \varphi}
$$

where $\omega_{0}$ is the angular frequency of the first spectral component. Note that $\varphi$ can be seen as the curvature of the parabola of the phases and that when $\varphi=2 \pi / N$ and $h(n)=1$ for $0 \leq n \leq N-1$ (flat spectrum), the phases of the modes match the Newman phases. In the frame rotating at $\omega_{0}$ and introducing $\theta=\omega_{s} t$, the electric field rewrites:

$$
E_{\varphi}(\theta)=E_{0} \sum_{n} h(n) e^{-i n \theta} e^{i \frac{n^{2}}{2} \varphi}
$$

The Poisson summation formula applied to the expression of the intensity $I_{\varphi}(\theta)=E_{\varphi}(\theta) E_{\varphi}^{*}(\theta)=\left|E_{\varphi}(\theta)\right|^{2}$ yields [19]: 

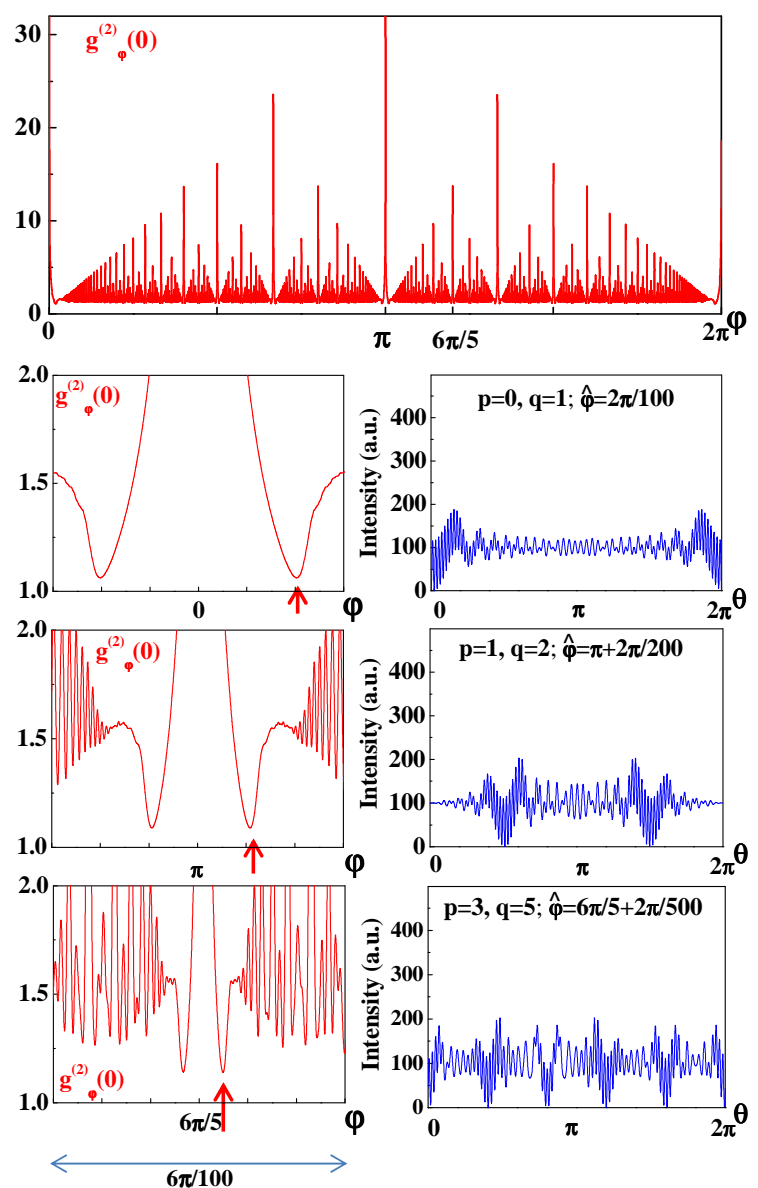

FIG. 3. Top: Plot of $g_{\varphi}^{(2)}(0)$ as a function of $\varphi$ in the case of $N=100$ modes with equal amplitude. Bottom left: zoom of $g_{\varphi}^{(2)}(0)$ in the vicinity of $\varphi / 2 \pi=p / q$ with $p / q=0,1 / 2$ and $3 / 5$ from top to bottom. The horizontal span is $6 \pi / 100$. The dips correspond to the generalized Newman phases $\hat{\varphi}=2 \pi(p / q \pm$ $1 / q N)$. The corresponding intensities $I_{\hat{\varphi}}(\theta)$ are plotted on the right column and the values of $g_{\hat{\varphi}}^{(2)}(0)$ are equal to $1.06,1.09$ and 1.15 respectively (top to bottom).

$$
I_{\varphi}(\theta)=I_{0} \sum_{n, m} H(m, n-m \varphi / 2 \pi) e^{-i m \theta} e^{i \frac{m^{2}}{2} \varphi}
$$

where $I_{0}=\left|E_{0}\right|^{2}$ and $H\left(x_{1}, x_{2}\right)=\int_{0}^{N} h(y+$ $\left.x_{1}\right) h(y) e^{-2 i \pi y x_{2}} d y$. In the two-dimensional plane, $H\left(x_{1}, x_{2}\right)$ is a function centered at the origin and the widths of $H$ along $x_{1}$ and $x_{2}$ scale respectively as $N$ and $1 / N$.

The degree of second-order coherence at zero delay is:

$$
g_{\varphi}^{(2)}(0)=\frac{<I_{\varphi}(\theta)^{2}>}{<I_{\varphi}(\theta)>^{2}}
$$

Since $I_{\varphi}(\theta)$ is real we have in the general case: $<I_{\varphi}(\theta)^{2}>=I_{0}^{2} \sum_{m, n, l} H(m, n-m \varphi / 2 \pi) H^{*}(m, n+$ $l-m \varphi / 2 \pi)$. Because the extension of $H$ along the second coordinate scales as $1 / N$, only the term $l=$ 0 has a significant contribution and $\left.<I_{\varphi}(\theta)^{2}\right\rangle=$ $I_{0}^{2} \sum_{m, n}|H(m, n-m \varphi / 2 \pi)|^{2}$. Moreover: $<I_{\varphi}(\theta)>^{2}=$ $I_{0}^{2}\left(\sum_{n} H(0, n)\right)^{2}=I_{0}^{2} H(0,0)^{2}$.

Interestingly it can be shown that $g_{\varphi}^{(2)}(0)$ (as a function of $\varphi$ ) tends to the so-called Thomae's function, i.e. $T(\varphi)=1 / q$ when $\varphi=2 \pi p / q$ where $q<N$ (Fig. 3) [19]. Note that $g_{\varphi}^{(2)}(0)$ shows dips not only in the vicinity of $\varphi=2 k \pi$ (more precisely at $\varphi=2 k \pi \pm 2 \pi / N$ which corresponds to the Newman phases) but also in the vicinity of any rational value of $\varphi / 2 \pi$. In the following we prove that at these locations $g_{\varphi}^{(2)}(0)$ tends to unity when $N$ becomes large.

We define $\hat{\varphi}=2 \pi(p / q \pm 1 / q N)$. Again Newman phases correspond to the specific case where $p=0$ and $q=1$. Defining $H_{0}=H(0,0)=\int h^{2}(y) d y$,

$$
g_{\hat{\varphi}}^{(2)}(0)=\frac{\sum_{m, n}\left|H\left(m, n-\frac{m p}{q} \mp \frac{m}{q N}\right)\right|^{2}}{H_{0}^{2}} .
$$

For the same reason as before the terms in the numerator having a significant contribution correspond to $m=k q, k$ integer and $n=\frac{m p}{q}$. Then $g_{\hat{\varphi}}^{(2)}(0)=$ $\frac{\sum_{k}\left|H\left(k q, \mp \frac{k}{N}\right)\right|^{2}}{H_{0}^{2}}$.

Again only the first terms (labeled by $k$ ) in the numerator contribute significantly. In the limit where $N$ is large $k q$ is much smaller than $N$ and $H\left(k q, \mp \frac{k}{N}\right) \approx H\left(0, \mp \frac{k}{N}\right)$. Using Parseval's theorem:

$$
g_{\hat{\varphi}}^{(2)}(0) \approx \frac{\sum_{k}\left|\int_{0}^{N} h^{2}(y) e^{ \pm i 2 \pi \frac{k}{N} y} d y\right|^{2}}{H_{0}^{2}}=\frac{N \int h^{4}(y) d y}{\left|\int h^{2}(y) d y\right|^{2}}
$$

which is equal to unity in the case of a flat spectrum. Therefore the intensity fluctuations tend to vanish when the phases of the modes are equal to $\hat{\varphi}=2 \pi(p / q \pm 1 / q N)$, which constitutes a generalization of the Newman phases. In the following these phases are called generalized Newman phases.

Generation of a comb of optical frequencies with quadratic phases in FSF lasers We now turn to the experimental implementation of quiet (or near constant intensity) broadband light. In optics, the generation of combs of optical frequencies with identical (or linear) phases is routinely achieved in mode-locked lasers. However a comb of optical frequencies with quadratically varying phase is more challenging to achieve since it requires a dispersion between adjacent modes linearly increasing with $n$, implying a very large group velocity dispersion. An interesting possibility is offered by linearly chirped fiber Bragg gratings but is restricted to combs with large frequency spacings [20]. However it turns out 


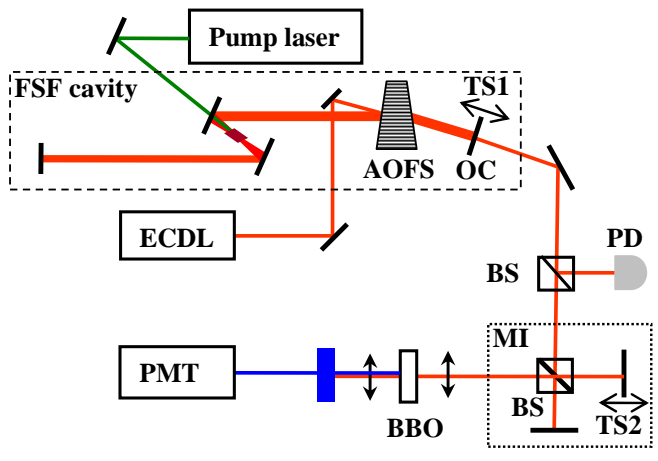

FIG. 4. Sketch of the experimental set-up (see text). The output coupler (OC) of the injection-seeded FSF Ti:Sa cavity is mounted on a translation stage (TS1). A Michelson interferometer (MI) and a BBO crystal tuned for SHG at 780 $\mathrm{nm}$ enable to record the IAC. BS, PD and PMT stand respectively for beamsplitter, photodiode and photomultiplier tube.

that a CW injection-seeded frequency shifted feedback (FSF) laser is a simple and flexible solution for the generation of an optical frequency comb with quadratic phases [21]. Recall that a FSF laser cavity is closed of the +1 (or -1) diffraction order of an acousto-optic frequency shifter (AOFS). Each time a photon makes a roundtrip in a (linear) FSF cavity, its frequency is shifted by twice the frequency of the acoustic wave $f_{s} / 2$. When operating without an external seeding, the resulting spectrum is modeless and the resulting laser field is similar in terms of statistical properties, to a chaotic field (i.e. $\left.g^{(2)}(0)=2\right)$ [22]. When a FSF cavity is seeded with a single frequency laser the resulting spectrum consists in a comb of optical frequencies with a frequency spacing equal to $f_{s}$. Interestingly it can be shown that the phases of the modes of the comb evolve quadratically with $n$ and can be written as: $\varphi_{n}=\pi n(n+1) \frac{f_{s}}{f_{c}}$ where $f_{c}$ is the cavity free spectral range defined as the inverse of the roundtrip time in the cavity $[19,21]$. The linear term in $\varphi_{n}$ results in a simple temporal shift. The possibility to tune the curvature of the phase parabola by adjusting $f_{s}$ or $f_{c}$ leads to the demonstration of ultrahigh repetition rates by a temporal fractional Talbot effect: when the ratio $f_{s} / f_{c}$ is set as the rational $p / q$ with $q \ll N$ the laser emits Fourier transform-limited pulses at a repetition rate equal to $p f_{c}=q f_{s}$ [21]. On the contrary adjusting the curvature of the phases parabola according to the generalized Newman phases leads to the possibility of using this laser architecture to generate quiet (i.e. near constant intensity) broadband light.

Experimental demonstration A linear Z-shape Ti:Sa laser cavity is closed on the +1 diffraction order of an acousto-optic frequency shifter driven at $40 \mathrm{MHz}\left(f_{s}=\right.$ $80 \mathrm{MHz}$ ). The optical roundtrip length of the FSF cavity is about $3 \mathrm{~m}$, resulting in a cavity free spectral range $f_{c}$
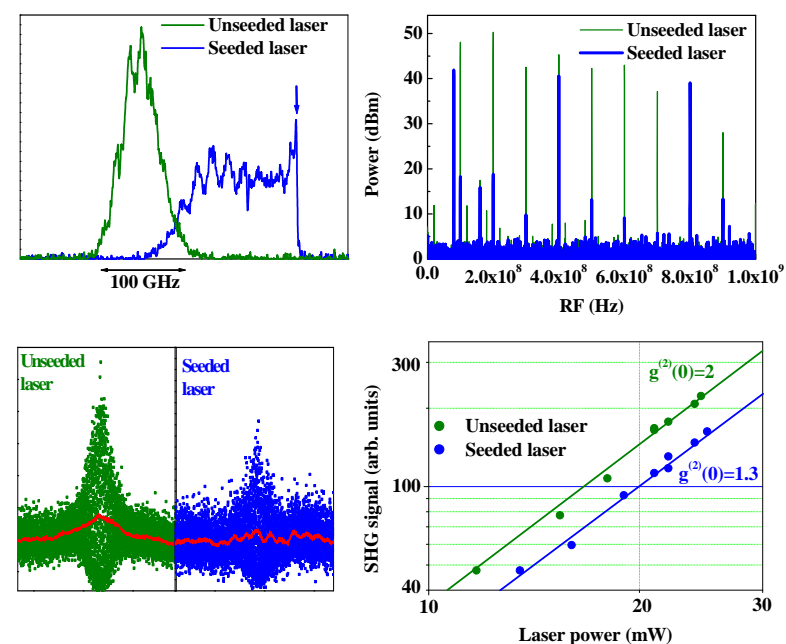

FIG. 5. Experimental comparison of the FSF laser operating without (in green) and with external seeding (in blue) when the cavity length is adjusted according to the generalized Newman phase $\hat{\varphi}=2 \pi(p / q \pm 1 / q N)$ where $p=4, q=5$ and $N \approx 2500$ so as to minimize the intensity fluctuations. Top left: optical spectra of the laser without (i.e. modeless laser) and with external seeding. The sharp peak corresponds to the injection laser. Top right and bottom left: comparison of the $\mathrm{RF}$ spectra in the $0-1 \mathrm{GHz}$ range and the IAC of the laser without and with external seeding. Bottom right: corresponding SHG signals in the same experimental conditions. The solid lines are quadratic fits.

close to $100 \mathrm{MHz}$. The cavity output coupler is mounted on a translation stage for a slight tuning of the free spectral range (Fig. 4). The Ti:Sa crystal is pumped by a solid state laser at $532 \mathrm{~nm}$ with a power of $4.3 \mathrm{~W}$. The FSF cavity is injected by a single-mode Extended Cavity Diode Laser (ECDL) at $780 \mathrm{~nm}$ (25 mW power, 10 $\mathrm{kHz}$ linewidth). The power at the output of the injected FSF cavity reaches $30 \mathrm{~mW}$ and the spectrum is $200 \mathrm{GHz}$ wide. The beam is then sent into a Michelson interferometer and a BBO nonlinear crystal tuned for second harmonic generation at $780 \mathrm{~nm}$, to record the autocorrelation trace. When $f_{c}$ is exactly tuned to $100 \mathrm{MHz}$, the ratio $f_{s} / f_{c}$ is equal to $4 / 5$ and the laser emits pulses at the repetition rate of $400 \mathrm{MHz}$, according to the temporal fractional Talbot effect.

When the FSF laser operates without seeding it generates a $100 \mathrm{GHz}$ broadband modeless spectrum. When externally seeded, the resulting spectrum consists in a comb (not resolved by the spectrometer) of about $N=2500$ modes separated by $80 \mathrm{MHz}$. The optical spectra are plotted on Fig. 5. When the cavity free spectral range is adjusted to $f_{c}=99.990 \mathrm{MHz}$ so as to match the generalized Newman phases $\hat{\varphi}=2 \pi(4 / 5+1 / 12500)$ the intensity spectrum shows significantly less noise as compared to the modeless laser (Fig. 5, top right) and the interferometric autocorrelation trace averaged over the fringes 


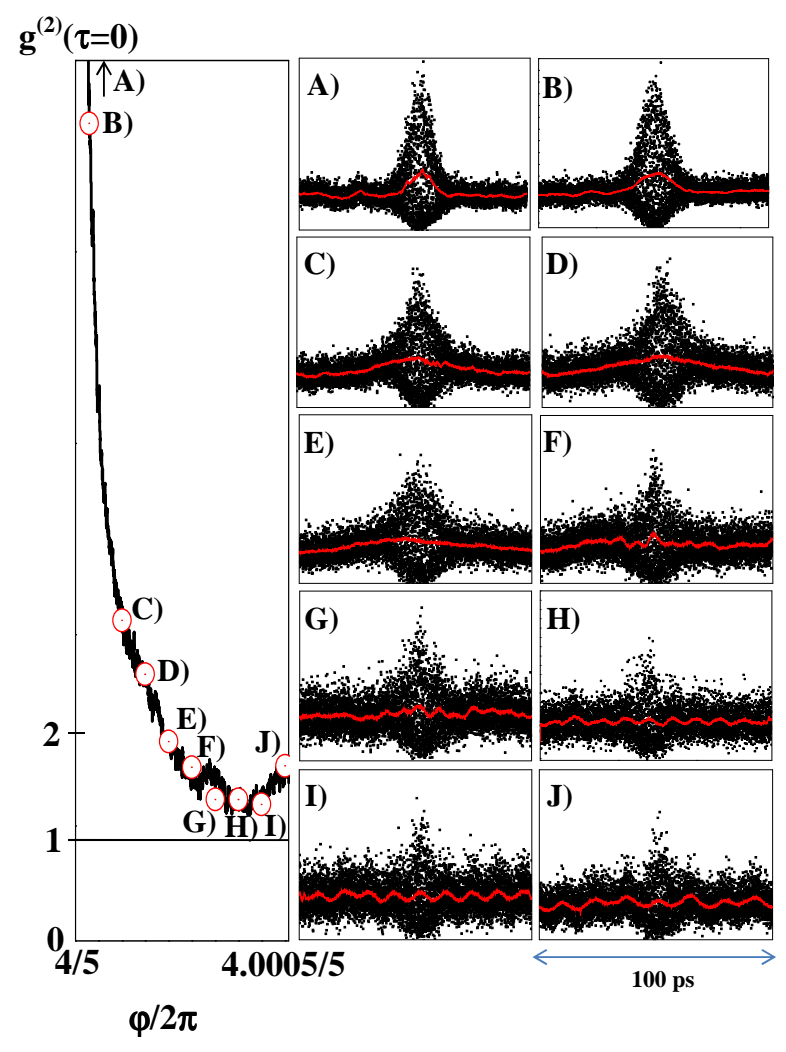

FIG. 6. Experimental IAC traces of the injection seeded laser in the vicinity of the minimum of fluctuations. Left: the vertical scale $\left(g^{(2)}(\tau=0)\right)$ has been adjusted by comparison to the unseeded laser.

shows a flat response, contrary to the trace of the modeless laser which displays a 3:2 peak-to-baseline ratio, as predicted on Fig. 2 (Fig. 5, bottom left). To infer a precise value of the $g^{(2)}(0)$ of the CW injection-seeded FSF laser we proceed as follows: the mobile arm of the interferometer is blocked and the intensity of the SHG signal is detected for different intensities of the pump laser, in the cases of both the modeless laser and the seeded laser. The experimental set-up is exactly the same in both cases. Since the modeless laser displays the statistical properties of a chaotic source (i.e. $\left.g^{(2)}(0)=2\right)$ one deduces that for the FSF laser $g^{(2)}(0)=1.3$ (Fig. 5 bottom right), which demonstrates intensity fluctuations strongly reduced compared to a chaotic light source. Note however that this value is higher than a constant intensity light that would lead to $g^{(2)}(0)=1$. This mainly comes from the fact that the experimental spectrum at the output of the seeded FSF laser is not flat, which leads to some discrepancy compared to the previous calculations. According to the experimental spectrum (Fig. 5 , top left) the expected value of $g_{\hat{\varphi}}^{(2)}(0)=N \frac{\int h^{4}(y) d y}{\left|\int h^{2}(y) d y\right|^{2}}$ is found to be 1.22 which is closer to the experimental value. Finally we record successively the interferomet- ric autocorrelation traces of the (seeded) FSF laser while scanning the cavity length in the vicinity of $\varphi=2 \pi \times 4 / 5$, and observe the transition from maximal to minimal fluctuations (fig. 6, A) to $\mathrm{H}$ )). When $\varphi$ exceeds $\hat{\varphi}$ (fig. $6, \mathrm{H}$ ) to J)) the average autocorrelation trace shows a modulation which is the signature that $\varphi / 2 \pi$ approaches other rational quantities $p^{\prime} / q^{\prime}$ (with $q^{\prime}<N$ ).

Finally it is important to justify that this experiment satisfies indeed the conditions of validity of the transposition, in the optical domain, of the reduction of the intensity fluctuations by a specific engineering of the phases. First quantum noise is negligible in the present case: the average power per frequency component is about $10 \mu \mathrm{W}$ and the typical measurement time scale is in the $\mu$ s range, which makes the signal to (quantum) noise ratio close to $10^{4}$. Second despite the lack of dynamical studies on CW injection-seeding FSF lasers, it is reasonable to assume that the relative amplitude of the individual frequencies remain constant at the measurement time scale. This assumption is supported by heterodyne measurements between the seed laser and the laser field at the output of the FSF cavity, which show that the intensity of the first frequency-shifted modes remain constant. Moreover the optical spectrum measured with a $\mathrm{GHz}$ resolution shows no fluctuations in shape or intensity down to the ms time scale. Finally the intrinsic dynamics (i.e. relaxation oscillations) of the homogeneous gain medium applies identically to the whole spectrum and should not affect significantly the relative amplitude of the individual frequency components. Therefore our system consisting in a comb of optical frequencies with quadratic relative phases constitutes a convincing optical analogue of its radiowave or acoustic counterparts demonstrated so far.

Conclusion We have demonstrated the possibility to lower significantly the intensity fluctuations of a broadband light source resulting from intermodal beating, by a quadratic adjustment of the phases of the modes. Our demonstration applies to near-flat combs of optical frequencies, with constant relative amplitudes and negligible quantum noise. We have generalized the Newman phases and demonstrated that for many other values of the curvature of the phase parabola, the intensity fluctuations of a flat spectrum of equidistant frequencies tend to cancel in the limit of a large number of modes. This general result could be applied to other fields of wave physics, including wireless communications, OFDM, acoustics, hydrodynamics and even quantum mechanics. Then we have applied this concept to optics and implemented a laser generating about 2500 modes arranged according to these generalized Newman phases and displaying intensity fluctuations reduced down to $g^{(2)}(0)=1.3$ (or $\left.C=0.54\right)$, that is significantly lower compared to a chaotic light source for which $g^{(2)}(0)=2$ and $C=1$. This demonstration could find applications in DWDM fiber networks where intensity fluctuations need to be limited to avoid non-linear effects responsible for 
channel cross talk. Moreover it could bring a solution to the distortion of signals at the output of optical amplifiers. Finally the injection seeded FSF laser constitutes a unique broadband light source with constant spectrum, constant average power and photon statistics easily tunable from super poissonian to poissonian.

Acknowledgements The authors acknowledge the Réseau FEMTO of the CNRS for its financial support.

* hugues.guilletdechatellus@ujf-grenoble.fr

[1] Y. Rahmatallah, S. Mohan, Peak-To-Average Power Ratio Reduction in OFDM Systems: a Survey And Taxonomy, IEEE Communications Surveys and Tutorials, 15, 4, 1567(2013).

[2] E. Van der Ouderaa, J. Schoukens, J. Renneboog, Peak Factor Minimization of Input and Output Signals of Linear Systems, IEEE Transactions on Instrumentation and Measurement 37, 2, 207(1988).

[3] W. M. Hartmann, J. Pumplin, Periodic signals with minimal power fluctuations, J. Acoust. Soc. Am. 90, 1986 (1991).

[4] M. Friese, Multitone Signals with Low Crest Factor, IEEE Transactions on Communications, 45, 10, 1338 (1997).

[5] M. Solomou, C. Evans, D. Rees, Crest Factor Minimization in the Frequency Domain, IEEE Transactions on Instrumentation and Measurement, 51, 4, 859 (2002).

[6] S. Boyd, Multitone Signals with Low Crest Factor, IEEE Transactions on Circuits and Systems, CAS-33, 10, 1018 (1986).

[7] M. R. Schroeder, "Number Theory in Science and Communications", 5th ed. (Springer, Berlin Heidelberg 2009), Chap. 28.

[8] M. R. Schroeder, Synthesis of Low-Peak-Factor Signals and Binary Sequences with Low Autocorrelation, IEEE Transactions on Information Theory, 16, 85 (1970).

[9] J. Pumplin, Low-noise noise, J. Acoust. Soc. Am. 78, 1, 100 (1985).
[10] D. J. Newman, An L1 extremal problem for polynomials, Proc. Am. Math. Soc. 16, 1287 (1965).

[11] D. R. Gimlin, C. R. Patisaul, On minimizing the Peak-toAverage Power Ratio for the sum of N Sinusoids, IEEE Transactions on Communications, 40, 631 (1993).

[12] W. M. Hartmann, J. Pumplin, Noise power fluctuations and the masking of sine signals, J. Acoust. Soc. Am. 83, 3, 2277 (1988).

[13] A. Kohlrausch, S. van de Par, On the use of specific signal types in hearing research, Oscillations, Waves and Interactions 37-71 (T. Kurz, U. Parlitz, U. Kaatze eds.) (2007).

[14] Interestingly many applications of the PAR problem in acoustics concern its spatial counterpart, that is the engineering of isotropic sound diffusors. Quadratic Residue Diffusers based on Schroeder phases are routinely used in concert halls and recording studios. See M. R. Schroeder, "Number Theory in Science and Communications", 5th ed. (Springer, Berlin Heidelberg 2009), Chap. 15.

[15] A. B. Wright, B. Xie, A. Karthikeyan, A comparison of white test signals used in active sound cancellation, Appl. Acoustics, 59, 337 (2000).

[16] E. Mozeson, N. Levanon, Multicarrier radar signals with low peak-to-mean enveloppe power ratio, IEE Proc. Radar Sonar Navig. 150, 2, 71 (2003).

[17] V. Aue, G. P. Fettweis, Multi-carrier spread spectrum modulation with reduced dynamic range, Proc. 46th IEEE Veh. Technol. Conf. 914 (1996).

[18] H.-A. Bachor, T. C. Ralph, "A Guide to Experiments in Quantum Optics", 2nd ed. (Wiley-VCH, Weinheim 2004), Chap. 2.

[19] H. Guillet de Chatellus, E. Lacot, W. Glastre, O. Jacquin, O. Hugon, Theory of Talbot lasers, Phys. Rev. A 88, 033828 (2013).

[20] J. Azaña, M. A. Muriel, Temporal Self-Imaging Effects: Theory and Application for Multiplying Pulse Repetition Rates IEEE. J. Sel. Top. in Quant. Elec., 7, 728 (2001).

[21] H. Guillet de Chatellus, O. Jacquin, O. Hugon, W. Glastre, E. Lacot, J. Marklof, Generation of ultrahigh and tunable repetition rates in $\mathrm{CW}$ injection-seeded frequency-shifted feedback lasers, Opt. Exp. 21, 1315065 (2013).

[22] H. Guillet de Chatellus, J.-P. Pique, Statistical properties of frequency-shifted feedback lasers, Opt. Comm. 283, 1971 (2010). 